\title{
Adventures in heparan sulfate degradation
}

\section{Liang Wu${ }^{1}$, Zachary Armstrong ${ }^{2}$, Casper de Boer ${ }^{3}$, Vincent $\mathrm{Lit}^{3}$, Gijs Ruijgrok ${ }^{3}$, Uri Barash ${ }^{4}$, llanit Boyango ${ }^{4}$, Neta Ilan $^{4}$, Israel Vlodavsky ${ }^{4}$, Hermen S Overkleeft ${ }^{3}$, Gideon J Davies ${ }^{2}$}

\author{
${ }^{1}$ The Rosalind Franklin Institute, Didcot, OX11 OFA, United Kingdom \\ ${ }^{2}$ Department of Chemistry, University of York, York, YO10 5DD, United Kingdom \\ ${ }^{3}$ Department of Bio-organic Synthesis, Leiden Institute of Chemistry, Leiden University, Einsteinweg 55, 2333 CC Leiden, The \\ Netherlands \\ 4Technion Integrated Cancer Center (TICC), The Bruce Rappaport Faculty of Medicine, Technion, Haifa 31096, Israel \\ liang.wu@rfi.ac.uk
}

Heparan sulfate (HS) is a ubiquitous glycosaminoglycan component of the extracellular matrix (ECM), which facilitates important structural and signalling interactions between cells and their surroundings. The principal enzyme responsible for extracellular HS breakdown is heparanase (HPSE), an endo-glucuronidase of the CAZy GH79 family. Whilst normal HPSE activity is essential for HS processing, excessive HPSE overexpression weakens HS networks in the ECM, leading to increased cell mobility and release of growth factors stored by HS. Thus HPSE is an oncogene whose overexpression promotes metastasis in a range of cancers.

In this talk, I will give an overview of our work in this area over the last few years, covering our initial structural investigations into the molecular basis of HPSE activity, the development of probes to visualize HPSE in tissues, and most recently, the structure guided rational design of HPSE inhibitors as anti-metastatic agents.

1. L. Wu, C. M. Viola et al (2015), Nat. Struct. Mol. Biol. (22) 1016-1022

2. L. Wu, J. Jiang, Y. Jin et al (2017), Nat. Chem. Biol. (13) 867-873 